\title{
Trotz Krise: Tariflicher Gesundheitsschutz im Sozial- und Erziehungsdienst
}

\author{
Sylvia Skrabs \\ Alexander Wegner
}

\section{ARBEITS- UND GESUNDHEITS- SCHUTZ IM TARIFVERTRAG}

Den Arbeits- und Gesundheitsschutz (AGS) tarifvertraglich zu verhandeln, ist bislang eher die Ausnahme. Denn es gibt seit 1996 ein Arbeitsschutzgesetz, das die durchzuführenden Maßnahmen bündelt und eine ganzheitliche Gefährdungsbeurteilung für jeden einzelnen Arbeitsplatz vorschreibt. Damit werden den Belegschaften und ihren Vertretungen im Bereich der Betriebsverfassung weitgehende Mitbestimmungsrechte eingeräumt. Im Unterschied dazu sind diese Möglichkeiten im Personalvertretungsrecht nicht in diesem Umfang vorhanden.

Wie sehr der hohe Anspruch des Arbeitsschutzgesetzes die Wirklichkeit verfehlt - und zwar sowohl hinsichtlich der Belastungssituation für die Beschäftigten als auch mit Blick auf die Umsetzung gesetzlicher AGS-Vorgaben - zeigt der hier im Fokus stehende Sozial- und Erziehungsdienst. Dessen Defizite langfristig zurückzudrängen, war - neben der wiederkehrenden Lohnfrage - der Anspruch der Tarifverhandlungen 2009: mit einem kleinen Erfolg für den Gesundheitsschutz, wie in diesem Beitrag deutlich wird.

\section{MOTIVATION FÜR EINEN NEUEN WEG}

Drei zentrale Motive waren wichtig für diese Stoßrichtung und verdeutlichen die Strategie von ver.di als Verhandlungsführerin für die Beschäftigteninteressen:

(1) Schaffung guter Arbeitsbedingungen, um die Gesundheit der Beschäftigten langfristig zu erhalten: Beschäftigte, die Achtung im Unternehmen erfahren, sich im Unternehmen wohl fühlen, selbst Entscheidungen treffen und eigenverantwortlich arbeiten können, ohne dass ihre Gesundheit verschlissen wird, werden mit Kreativität, Aufgeschlossenheit und einer positiven Einstellung ihnen anvertrauten Menschen,
Bürgerinnen und Bürgern, Patientinnen und Patienten gegenübertreten können. Das belegen viele arbeitswissenschaftliche Untersuchungen und das war Maßstab für diegesundheitspolitischen Forderungen im Tarifvertrag. Deren Verwirklichung setzt jedoch voraus, den Beschäftigten Würde, Respekt und Anerkennung zu zollen und Belastungen, die zu Gesundheitsgefährdungen führen und das Wohlbefinden einschränken, zu bekämpfen. Als geeignetes Instrument für diese Zielsetzung wurde deshalb die vom Arbeitsschutzgesetz vorgeschriebene Gefährdungsbeurteilung in den Vordergrund gerückt.

(2) Die Verwirklichung der „Konsumenten“Forderungen im Bereich der sozialen Betreuungsdienstleistungen: Hier sind an erster Stelle natürlich die Eltern zu nennen, die seit Jahren auf die problematischen Zustände in Kindergärten und anderen Erziehungseinrichtungen hinweisen. Aber es geht auch um die Kinder und die anderen zu betreuenden Gruppen, die unter den schwierigen Arbeitsbedingungen der Sozialarbeiterinnen und Sozialarbeiter, der Erzieherinnen und Erzieher in Form von mangelnder Berücksichtigung ihrer Wünsche und Bedürfnisse zu leiden hatten.

(3) Ein Zeichen gegen Sozialabbau in der Zeit der sich anbahnenden Finanz- und Wirtschaftskrise setzen: Es sollte außerdem gezeigt werden, dass vermeintlich weiche Themen wie die Gesundheit der Beschäftigten auch in harte Tarifverhandlungen eingebracht werden können. Gerade in einer Krisenzeit muss die Frage gestellt werden, welche Bedeutung die Menschen und ihre Arbeitsbedingungen für die Gesellschaft haben. Es sollte auch Ausdruck für die Wertschätzung der geleisteten Arbeit und eingebrachten Motivation für diese besondere Form der personenbezogenen, sozialen Dienstleistungen sein, die in ihrer wirtschaftlichen Bedeutung künftig weiter wachsen werden. Gesellschaftliche Auf- wertung dieser Arbeit wird somit auch zu einer Krisenbegegnungsstrategie, indem stärker nachgefragte Dienstleistungen als Beschäftigungsfelder attraktiver gemacht werden. Die Krise und knappe (öffentliche) Kassen sind gerade deshalb kein Hinderungsgrund für die Thematisierung von Gesundheit, weil in dieser Zeit die Weichen neu gestellt werden müssen. Zudem wurden die Beschäftigten ja bereits in der Zeit von Wirtschaftswachstum und Boom vertröstet, mit dem Hinweis, dass zunächst erst einmal das finanzielle Polster für Handlungsfähigkeit bei Schaffung günstigerer Arbeitsbedingungen angelegt werden müsse. Faktisch werden die Beschäftigtenforderungen ignoriert, wenn je nach Wirtschaftslage auf das Ende der konjunkturellen Talfahrt oder das Ende eines Booms verwiesen wird.

Aus diesen drei Gründen heraus gilt es, neben der Bekämpfung von Lohndumping, Sozial- und Gesundheitsstandards stärker zu thematisieren und der tarifpolitischen Auseinandersetzung zugänglich zu machen. Wenn es gelingt, Gesundheitsschutz tarifpolitisch zu verankern, kommen wir auch dahin, schlechte Gesundheitsbedingungen, missbraucht als Wettbewerbsvorteil in einem umkämpften Markt, zurückzudrängen. Dabei gilt es, die Beschäftigten nicht nur in der tarifpolitischen Auseinandersetzung mit ins Boot zu holen, sondern

\footnotetext{
Sylvia Skrabs ist Tarifsekretärin in der Tarifpolitischen Grundsatzabteilung der ver.di-Bundesverwaltung. Arbeitsschwerpunkte: Arbeitszeitpolitik, tarifpolitische Gestaltungsmöglichkeiten zum Arbeitsund Gesundheitsschutz, Gute Arbeit. e-mail: sylvia.skrabs@verdi.de Alexander Wegner ist Tarifkoordinator im Fachbereich Gemeinden bei der ver.diBundesverwaltung. Arbeitsschwerpunkte: Tarifpolitik, Kommunen. e-mail: alexander.wegner@verdi.de
} 
auch Rechte zu verankern, dass sie bei der Umsetzung als Akteure in eigener Sache einbezogen bleiben.

\section{AUSGANGSLAGE IM SOZIAL- UND ERZIEHUNGSDIENST}

Beispielhaft wird hier auf die öffentlichkeitswirksamen Auseinandersetzungen im Sozial- und Erziehungsdienst - der sogenannte „KiTa-Streik“ - verwiesen, durch die für das Thema Gesundheit am Arbeitsplatz ein Tarifvertrag abgeschlossen werden konnte. Die Auseinandersetzung war notwendig, weil in diesem Bereich gesetzliche Normen und betriebliche Wirklichkeiten weit auseinanderklafften. Mit dem neuen Tarifvertrag erhalten die Beschäftigten dieser Branche eine Perspektive auf die Verbesserung ihrer Arbeitsbedingungen.

Die Tarifforderungen der Gewerkschaft und die ihnen zugrunde liegenden Motive sind auch eine Reaktion auf die Entwicklung der Arbeitsbedingungen der vergangenen Jahre in diesem Bereich. Seit Langem wird auf die besonderen Gesundheitsgefährdungen im Sozial- und Erziehungsdienst durch physische und psychische Belastungen hingewiesen. Insbesondere sind die erheblichen Lärmbelastungen, ergonomische Belastungsfaktoren (durch nicht erwachsenengerechtes Mobiliar) sowie eine Vielzahl psychischer Belastungen - hervorzuheben: Die Überlastung (im Sinne von Relation Arbeitskraft zu Arbeitsmenge) ist hierbei an erster Stelle zu nennen.

Dem dramatisch anwachsenden Fachkräftemangel und den steigenden Anforderungen an die Praxis der frühkindlichen Bildung - die Länder verabschieden zunehmend entsprechende gesetzliche Vorgaben - kann unter den gesundheitlichen Voraussetzungen nicht angemessen begegnet werden. Strategien zum „Älter werden im Beruf" beschäftigen die Einrichtungen und ihre Mitarbeiterinnen und Mitarbeiter heute; für die gewerkschaftliche Positionsbestimmung entsteht hier ein zentrales Thema und Handlungsfeld.

\section{VERHANDLUNGS- UND STREIKERGEBNISSE}

Mit einer Forderung zur Entgeltordnung (also zur Stellenbewertung) und einer Forderung zum betrieblichen Gesundheitsschutz konnten in dieser Tarifrunde die Arbeitsbedingungen umfassend thematisiert werden. Dadurch wurden die Positionen zu Stellenbewertungen auf ihren Kern, die Anforderungen an die Tätigkeit, fokussiert. Die Belastungsfaktoren konnten parallel dazu in den Blick genommen werden, ohne sie, quasi im Sinne von Schmerzensgeld, zum Diskussionsgegenstand im Kontext der Stellenbewertungen zu machen.

Es ist gelungen, trotz anfangs massivem Widerstand der Arbeitgeber, dieses Thema überhaupt zu verhandeln, im Tarifvertrag einen individuellen Anspruch der Beschäftigten auf die Durchführung einer Gefährdungsbeurteilung durchzusetzen. Dabei wurden sowohl ihre Beteiligung bei der Durchführung von Gefährdungsbeurteilungen als auch ihr Recht auf Unterrichtung über die Ergebnisse vereinbart. Letztlich ergänzt der Tarifvertrag die gesetzlich bereits vorliegenden Regelungen durch individuelle Rechtsansprüche. An einigen Stellen formuliert die Vereinbarung die Bedingungen für den Sozial- und Erziehungsdienst konkret aus, macht also Vorgaben z.B. für die Einrichtung einer Kommission und deren Geschäftsordnung.

Die Steuerung der Maßnahmen zum betrieblichen Gesundheitsschutz obliegt nun einer betrieblich einzurichtenden Kommission. Sie besteht zu einer Hälfte aus vom Personal- oder Betriebsrat bzw. aus Reihen der Beschäftigten und zur anderen Hälfte aus vom Arbeitgeber entsandten Vertretern. Vor dem Hintergrund gewerkschaftlicher Erfahrungen mit Prozessen des betrieblichen Gesundheitsschutzes besteht in der Kommission die Chance, die Möglichkeiten des Arbeitsschutzes besser nutzbar zu machen und eine breite Beschäftigtenbeteiligung abzusichern.

\section{BEWERTUNG UND HERAUS- FORDERUNGEN}

Die Tarifverhandlungen und die sie begleitenden Aktionen und Streiktage deckten die massiven Probleme auf, die das Berufsleben vieler Kolleginnen und Kollegen im Sozial- und Erziehungsdienst maßgeblich bestimmen. Auch darum wirkte die Tarifforderung von ver.di in hohem Maße mobilisierend und interessierte die Öffentlichkeit. Den entscheidenden Impuls gab jedoch das Engagement der Eltern, die sich darüber empörten, dass die gesundheits- beeinträchtigenden Arbeitsbedingungen Qualitätsprobleme zulasten von Kindern, anderen zu Betreuenden und Hilfsbedürftigen verursachen.

Der Streik wirkte aber auch deshalb, weil andere Branchen indirekt in Mitleidenschaft gezogen wurden und auf diese Weise von den Aktionen erfuhren. Vermittelt durch die mit dem Streik sympathisierend verbundenen Eltern, aber auch durch die Eltern, die sich gezwungen sahen, selbst eine Betreuung zu organisieren, waren viele Betriebe plötzlich betroffen, weil sie nicht mehr uneingeschränkt auf ihre Arbeitskräfte zugreifen konnten.

Der lange Atem von Erzieherinnen und Erziehern, Eltern und Gewerkschaften - der Tarifkonflikt erstreckte sich fast über ein halbes Jahr - und die öffentlichkeitswirksame Präsenz der Streikenden und Eltern brachten schließlich ein Einlenken der Arbeitgeber. Der Widerstand gerade gegen die gesundheitspolitischen Forderungen des Tarifvertrages wurde aufgegeben. Stattdessen wurde versucht, über die Ausgestaltung der betrieblichen Umsetzung der Gefährdungsbeurteilung - als das zentrale Instrument des Arbeitsund Gesundheitsschutzes - Kontrolle über den Prozess zu bekommen. Das Letztentscheidungsrecht der Geschäftsleitung ist Ergebnis dieser Verhandlungsstrategie. Für ver.di entscheidend war dabei, dass durch die innerbetriebliche Thematisierung von Gesundheitsbelastungen unter breiter Beteiligung von Beschäftigten, welche nun als Träger von Rechten teilhaben, eine Dynamik in Gang gesetzt wird, die es dem Arbeitgeber bei problematischen Gesundheitsbedingungen schwer macht, Maßnahmen zu verhindern.

Mit der Umsetzung des Tarifvertrags stellen sich aber auch einige Herausforderungen. Nicht nur bezogen auf die erforderlichen Schulungen der Personalräte und der Beschäftigten und die entsprechende Unterstützung, sondern auch bezogen auf die Nutzbarmachung der in den Betrieben gewonnenen ersten Erkenntnisse aus der Umsetzung des Tarifvertrages. Eine besondere Bedeutung kommt dabei der Untersuchung von Umgangsweisen mit psychischen Belastungen zu. Sie werden von den Fachkräften insbesondere mit der zu hohen Arbeitsmenge in Verbindung gebracht.

Im Arbeitsfeld Kindertageseinrichtungen berührt dies unmittelbar den durch Landesgesetze vorgeschriebenen Betreu- 
ungsschlüssel, d. h., wie viele Kinder eine Fachkraft maximal betreuen darf. Dass der bisher gültige Schlüssel zu einer Verschärfung der Gesundheitsbelastungen bei Erzieherinnen und Erziehern führt, haben viele Studien und Modellrechnungen nachgewiesen. Selbst die Europäische Kommission hat in ihren Empfehlungen für Bedingungen frühkindlicher Bildung anzustrebende Personalschlüssel formuliert, die fast eine Verdopplung des Personals in den Kindertageseinrichtungen erfordern würden. Die bestehenden Instrumente zur Identifizierung und Bemessung vorhandener psychischer Belastungen werden also in dem vorliegenden Kontext weiter zu entwickeln sein.

Gewerkschaften und Arbeitnehmervertretungen stehen dadurch vor der Herausforderung, sowohl die betriebliche Umsetzung von Verbesserungen der Arbeitsbedingungen zu befördern als auch die Ergebnisse auf abstrahierbare Sachverhalte zu prüfen, die überbetriebliche, gesetzliche Änderungserfordernisse belegen.
Die nun auf Grundlage des Tarifvertrages bevorstehenden Untersuchungen werden die Mängel der Einrichtungen in Ausstattung und baulicher Gestaltung aufdecken. Dies begründet einerseits den lokalen Änderungsbedarf und bedeutet bezogen auf physische Belastungen die Chance auf eine deutliche Verbesserung der Arbeitsbedingungen.

Andererseits werden diese Belastungsmessungen Handlungsbedarf auf der überbetrieblichen Ebene offenkundig machen, wie in der Diskussion um den Betreuungsschlüssel bereits angesprochen. Wenn die Untersuchungen psychischer Belastungen Ergebnisse hervorbringen, die, wie vermutet, eine Überlastung der Fachkräfte belegen, dann ist der Weg frei, wieder vor die Landesregierungen zu treten und bessere Bedingungen für die frühkindliche Bildung zu fordern.

Auch für diese Strategie werden dann, wie schon im zurückliegenden Arbeitskampf, die Eltern wichtige Partner sein, weil sie - wie die Erfahrungen aus dem
Streik 2009 gezeigt haben - den Konnex zwischen den Arbeitsbedingungen der Beschäftigten und den Lernbedingungen der Kinder nicht durch die Unterstützung eines Sparkurses aushebeln lassen wollen. Resümierend hinsichtlich der eingangs formulierten drei Motive für den 2009 erfolgreich geführten Arbeitskampf lässt sich also festhalten: Auf allen drei Ebenen Beschäftigte, Eltern/Kinder, Gesellschaft wurden die Gewerkschaften in ihrem Kurs bestätigt, neben den monetären Forderungen die Arbeits- und Gesundheitsbedingungen zum Thema von Tarifverträgen zu machen. Dabei hat die aufbrechende Krise, ausgehend von den Finanzmärkten, übergehend auf Realwirtschaft und öffentliche Haushalte, nicht zu einer Rücknahme dieser Forderungen geführt. Es hat den Anschein, dass sich mit der Thematisierung von Gesundheitsbedingungen der Arbeit in dieser Situation auch eine Rückbesinnung auf Überlegungen in der Gesellschaft verbinden lassen, wie wir künftig leben und arbeiten wollen. 